\title{
Time-Domain Orthogonal Finite-Element Reduction-Recovery Method for Electromagnetics-Based Analysis of Large-Scale Integrated Circuit and Package Problems
}

\author{
Duo Chen and Dan Jiao, Senior Member, IEEE
}

\begin{abstract}
A time-domain orthogonal finite-element reductionrecovery method is developed to overcome the large problem sizes encountered in the simulation of large-scale integrated-circuit and package problems. In this method, a set of orthogonal prism vector basis functions is developed. Based on this set of bases, an arbitrary 3-D multilayered system such as a combined package and die is reduced to a single-layer system with negligible computational cost. More importantly, the reduced single-layer system is diagonal and, hence, can be solved readily. From the solution of the reduced system, the solution of the other unknowns is recovered in linear complexity. The method entails no theoretical approximation. It applies to any arbitrarily shaped multilayer structure involving inhomogeneous materials or any structure that can be geometrically modeled by triangular prism elements. In addition, it permits nonlinear device modeling and broadband simulation within one run. Numerical and experimental results have demonstrated its accuracy and high capacity in simulating on-chip, package, and die-package interface problems.
\end{abstract}

Index Terms-Die-package cosimulation, electromagnetic simulation, finite-element methods, large scale, on-chip, package, time domain.

\section{INTRODUCTION}

$\mathbf{T}$ HE SCALING of supply voltages and the increased level of integration have conspired to make the analysis and design of microelectronic systems increasingly challenging. The impact of noise due to signal switching, die-package interaction, power management techniques, substrate coupling, etc., can be seen at all levels of a power delivery network, from chip to package to motherboard to the voltage regulator module. Prevailing circuit-based signal-integrity paradigms are reaching their limits of predictive accuracy when applied to the combined die-package problem. Thus, there is a critical

Manuscript received August 7, 2008; revised October 23, 2008. Current version published July 17, 2009. This work was supported in part by a Grant from Intel Corporation, by a Grant from the Office of Naval Research under Award N00014-06-1-0716, and by a Grant from the National Science Foundation under Award 0747578. This paper was recommended by Associate Editor R. Suaya.

D. Chen is with the On-Chip Electromagnetics Research Group, School of Electrical and Computer Engineering, Purdue University, West Lafayette, IN 47907 USA.

D. Jiao is with the School of Electrical and Computer Engineering, Purdue University, West Lafayette, IN 47907 USA (e-mail: djiao@purdue.edu).

Color versions of one or more of the figures in this paper are available online at http://ieeexplore.iee.org.

Digital Object Identifier 10.1109/TCAD.2009.2021010 need for the electromagnetics-based cosimulation of the die and package to control the global electrical interaction and optimize performance as an integrated system.

The cosimulation of the die and package results in numerical problems of ultra-large scale, requiring billions of parameters to describe them accurately. In order to address the large problem size, electromagnetic solutions have to scale favorably. In recent years, researchers have been developing electromagnetics-based computer-aided-design technologies amenable for integrated-circuit (IC) and packaging problems as can be seen from [1]-[19].

In [11] and [12], a time-domain layered finite-element reduction-recovery (LAFE-RR) method was developed for solving large-scale IC problems. This method can reduce the system matrix of size $O(N)$ rigorously to that of size $O(M)$ for any multilayered structure, with $N$ being the number of unknowns in the entire 3-D structure and $M$ being the number of unknowns in a single layer. Furthermore, the reduction from $O(N)$ to $O(M)$ is achieved without any computational cost via analytical means, and hence, the CPU and memory overheads are minimal. Moreover, the reduction preserves the sparsity of the original matrix, thus enabling an efficient computation of the reduced matrix. The solution of the $O(M)$ parameters generated by this method is the same as that of the $O(M)$ parameters in the original $O(N)$ equation. The method has been successfully applied to solve large-scale IC problems.

The remaining computational task in the LAFE-RR method is the solution of the reduced system. Although the sparsity is preserved in the reduction process, if the reduced system is large, solving it could be still challenging.

In this paper, an orthogonal finite-element reductionrecovery (OrFE-RR) method was developed to advance the LAFE-RR method to the level of simulating a combined diepackage system. In this method, a set of orthogonal prism vector basis functions is developed. These basis functions are complete. In addition, they enforce the tangential continuity of the electric field and the normal continuity of the electric flux across material interfaces. The advantage of this set of bases is three-fold. First, an arbitrary 3-D multilayered system such as a combined package and die can be reduced to a single-layer system with negligible computational cost. Second, the reduced single-layer system is diagonal and, hence, can be solved readily. Third, the solution of the unknowns in other layers 


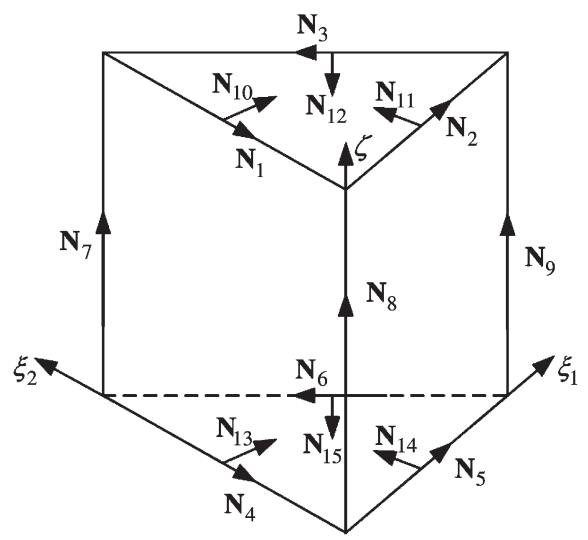

Fig. 1. Illustration of the orthogonal prism vector basis functions.

can be recovered in linear complexity. The method entails no approximation. It is applicable to arbitrarily shaped 3-D multilayer structures embedded in inhomogeneous media.

The remaining of this paper is organized as follows. In Section II, the orthogonal prism vector basis functions developed in this work are presented. In Section III, the time-domain OrFE-RR method is detailed. In Section IV, numerical and experimental results are given to demonstrate the accuracy and high capacity of the proposed method, and Section $\mathrm{V}$ relates to our conclusions.

\section{ORthogonal Prism Vector Basis Functions}

Based on our knowledge in 2-D orthogonal vector bases in a triangular element [20] and 3-D orthogonal vector bases in a tetrahedral element [21], the 3-D orthogonal vector bases in a prism element are developed as follows.

In each prism element, we construct fifteen vector bases from $\mathbf{N}_{1}$ to $\mathbf{N}_{15}$ as shown in Fig. 1. The degrees of freedom associated with bases $\mathbf{N}_{i}(i=1,2,3, \ldots, 6)$ are assigned on the upper and lower planes. Each of these bases is purely tangential to edge $i$ at the midpoint of edge $i$. They ensure the tangential field continuity across the element interface. They are given by

$$
\begin{aligned}
& \mathbf{N}_{i}=\zeta \mathbf{Z}_{i}, \quad i=1,2,3 \\
& \mathbf{N}_{i}=(1-\zeta) \mathbf{Z}_{i-3}, \quad i=4,5,6
\end{aligned}
$$

in which $\zeta$ is 0 on the lower plane and 1 on the upper plane, and vector bases $\mathbf{Z}_{i}$ are

$$
\mathbf{Z}_{i}=\left[\mathbf{W}_{i}^{e}-\sum_{m=1}^{3} \frac{\left\langle\mathbf{W}_{i}^{e}, \mathbf{B}_{m}^{e}\right\rangle}{\left\langle\mathbf{B}_{m}^{e}, \mathbf{B}_{m}^{e}\right\rangle} \mathbf{B}_{m}^{e}\right], \quad i=1,2,3
$$

where $\mathbf{W}_{i}^{e}(i=1,2,3)$ are the normalized edge basis functions in element $e$ given by [22, pp. 276-279]

$$
\begin{aligned}
\mathbf{W}_{1}^{e} & =l_{1}^{e}\left(\xi_{1} \nabla \xi_{2}-\xi_{2} \nabla \xi_{1}\right) \\
\mathbf{W}_{2}^{e} & =l_{2}^{e}\left(\xi_{2} \nabla \xi_{3}-\xi_{3} \nabla \xi_{2}\right) \\
\mathbf{W}_{3}^{e} & =l_{3}^{e}\left(\xi_{3} \nabla \xi_{1}-\xi_{1} \nabla \xi_{3}\right)
\end{aligned}
$$

in which $l_{i}^{e}(i=1,2,3)$ is the length of edge $i$ in element $e$, $\xi_{i}(i=1,2,3)$ are the area coordinates [22, pp. 95-97], and
$\mathbf{B}_{i}^{e}(i=1,2,3)$ are the supplemental vector basis functions given by

$$
\mathbf{B}_{1}^{e}=\xi_{1} \xi_{2} \hat{n}_{1} \quad \mathbf{B}_{2}^{e}=\xi_{2} \xi_{3} \hat{n}_{2} \quad \mathbf{B}_{3}^{e}=\xi_{3} \xi_{1} \hat{n}_{3}
$$

in which $\hat{n}_{i}$ denotes the unit vector that is normal to edge $i$.

The degrees of freedom associated with bases $\mathbf{N}_{i}(i=$ $7,8,9)$ are assigned along the vertical edges of a prism element. They also guarantee the tangential field continuity across the element interface. These bases are given by

$$
\mathbf{N}_{7}=\xi_{1} \nabla \zeta \quad \mathbf{N}_{8}=\xi_{2} \nabla \zeta \quad \mathbf{N}_{9}=\xi_{3} \nabla \zeta .
$$

From (1) and (2), it can be seen that the normal components of bases $\mathbf{N}_{i}(i=1,2,3, \ldots, 6)$ vanish at the midpoints of the element edges on the upper and lower triangular elements shown in Fig. 1. Hence, they form an incomplete set of bases, i.e., they cannot be used to represent fields that have nonvanishing normal components at the midpoints of the element edges. To make them complete, bases $\mathbf{N}_{i}(i=10,11,12, \ldots, 15)$ are constructed to be complementary to bases $\mathbf{N}_{i}(i=1,2,3, \ldots, 6)$. They supplement the normal components of the electric fields. In addition, they are associated with each individual element, i.e., not shared by elements, hence allowing for the normal discontinuity of $\mathbf{E}$ and thereby ensuring the normal continuity of the electric flux across element interfaces in the variational process of a finite-element procedure. These bases are given by

$$
\begin{aligned}
& \mathbf{N}_{i}=\zeta \mathbf{B}_{i-9}^{e}, \quad i=10,11,12 \\
& \mathbf{N}_{i}=(1-\zeta) \mathbf{B}_{i-12}^{e}, \quad i=13,14,15 .
\end{aligned}
$$

Defining a numerical integration on the upper and lower planes as

$$
\left\langle\mathbf{N}_{i}^{e}, \mathbf{N}_{j}^{e}\right\rangle_{S}=\sum_{l=1}^{3} \alpha_{l} \mathbf{N}_{i}^{e}\left(m_{l}\right) \cdot \mathbf{N}_{j}^{e}\left(m_{l}\right)
$$

where $m_{l}$ denotes the midpoint of edge $l$ and $\alpha_{l}$ is the coefficient chosen such that the numerical integration is at least second-order accurate (generally chosen as $1 / 3$ in our implementation), we obtain

$$
\begin{aligned}
& \left\langle\mathbf{N}_{i}^{e}, \mathbf{N}_{j}^{e}\right\rangle_{V}=0, \quad i=1,2,3,10,11,12 ; j=1,2,3,10,11,12 ; i \neq j \\
& \left\langle\mathbf{N}_{i}^{e}, \mathbf{N}_{j}^{e}\right\rangle_{V}=0, \quad i=4,5,6,13,14,15 ; j=4,5,6,13,14,15 ; i \neq j \\
& \left\langle\mathbf{N}_{i}^{e}, \mathbf{N}_{j}^{e}\right\rangle_{V}=0, \quad i=1,2,3,10,11,12 ; j=4,5,6,13,14,15 ; j \neq i+3
\end{aligned}
$$

i.e., basis functions $\mathbf{N}_{i}(i=1,2,3,10,11,12)$ are orthogonal to each other, $\mathbf{N}_{i}(i=4,5,6,13,14,15)$ are orthogonal to each other, and these two sets are also mutually orthogonal to each other. In addition, because the bases on the lower and upper planes are perpendicular to the vector bases along the vertical edges, we have

$$
\begin{aligned}
\left\langle\mathbf{N}_{i}^{e}, \mathbf{N}_{j}^{e}\right\rangle_{V} & =0, \quad i=1,2,3,10,11,12 ; j=7-9 \\
\left\langle\mathbf{N}_{i}^{e}, \mathbf{N}_{j}^{e}\right\rangle_{V}=0, & i=4,5,6,13,14,15 ; j=7-9
\end{aligned}
$$

i.e., basis functions $\mathbf{N}_{i}(i=1,2,3,10,11,12)$ and $\mathbf{N}_{i}(i=$ $4,5,6,13,14,15)$ are orthogonal to basis functions $\mathbf{N}_{i}(i=$ $7,8,9)$. 


\section{Time-Domain OrFE-RR Method}

In this section, first, a time-domain finite-element framework is outlined. The proposed OrFE-RR method is then detailed, which includes the reduction from a 3-D layered system to a single-layer one that is diagonal in negligible computational cost, the recovery of other unknowns in linear complexity, and the performance analysis.

\section{A. Time-Domain Finite-Element Framework}

Compared with other time-domain computational electromagnetic methods such as finite-difference time-domain-based methods and time-domain integral-equation-based methods, a time-domain finite-element method (TDFEM) [23] deals with sparse matrices as well as possessing increased capability in handling irregular geometries and arbitrary inhomogeneity. In this section, we outline the basic numerical scheme of the TDFEM.

The electric field $\mathbf{E}$ inside an IC and package problem satisfies the second-order vector wave equation

$$
\begin{aligned}
\nabla \times\left[\mu_{r}^{-1} \nabla \times \mathbf{E}(\mathbf{r}, t)\right] & +\mu_{0} \varepsilon \partial_{t}^{2} \mathbf{E}(\mathbf{r}, t) \\
& +\mu_{0} \sigma \partial_{t} \mathbf{E}(\mathbf{r}, t)=-\mu_{0} \partial_{t} \mathbf{J}(\mathbf{r}, t)
\end{aligned}
$$

subject to certain boundary conditions. In (10), $\mu_{r}$ is the relative permeability, $\mu_{0}$ is the free-space permeability, $\varepsilon$ is the permittivity, and $\sigma$ is the conductivity. The time-domain finite-element solution of (10) results in the following system of ordinary differential equations:

$$
\mathbf{T} \frac{d^{2} u}{d t^{2}}+(\mathbf{R}+\mathbf{Q}) \frac{d u}{d t}+\mathbf{S} u+f=0
$$

in which $\mathbf{T}, \mathbf{R}, \mathbf{Q}$, and $\mathbf{S}$ are square matrices, $u$ is the unknown field vector, and $f$ is the excitation vector. The elements of the matrices $\mathbf{T}, \mathbf{R}$, and $\mathbf{S}$ are given by

$$
\begin{aligned}
\mathbf{T}_{i j} & =\mu_{0} \varepsilon\left\langle\mathbf{N}_{i}, \mathbf{N}_{j}\right\rangle_{V} \\
\mathbf{R}_{i j} & =\mu_{0} \sigma\left\langle\mathbf{N}_{i}, \mathbf{N}_{j}\right\rangle_{V} \\
\mathbf{S}_{i j} & =\mu_{r}^{-1}\left\langle\nabla \times \mathbf{N}_{i}, \nabla \times \mathbf{N}_{j}\right\rangle_{V}
\end{aligned}
$$

in which $\mathbf{N}_{i}$ and $\mathbf{N}_{j}$ are the vector basis functions used to expand unknown fields, and $\langle\cdot, \cdot\rangle_{V}$ denotes volume integration. Matrix $\mathbf{Q}$ is related to the absorbing boundary condition. If the first-order absorbing boundary condition is used to truncate the computational domain, the elements of $\mathbf{Q}$ are given by

$$
\mathbf{Q}_{i j}=\frac{1}{c}\left\langle\hat{n} \times \mathbf{N}_{i}, \hat{n} \times \mathbf{N}_{j}\right\rangle_{\mathrm{S}}
$$

where $c$ is the speed of light, $\hat{n}$ represents a unit vector normal to the truncation boundary $S$ and pointing outward, and $\langle\cdot, \cdot\rangle_{S}$ denotes surface integration.

Adopting a central difference scheme to approximate the first- and second-order time derivatives in (11), we obtain

$$
\begin{aligned}
\tilde{\mathbf{T}} u^{n+1}=(2 \mathbf{T} & \left.-\Delta t^{2} \mathbf{S}\right) u^{n} \\
& +[0.5 \Delta t(\mathbf{R}+\mathbf{Q})-\mathbf{T}] u^{n-1}-\Delta t^{2} f^{n}
\end{aligned}
$$

in which

$$
\tilde{\mathbf{T}}=\mathbf{T}+0.5 \Delta t(\mathbf{R}+\mathbf{Q})
$$

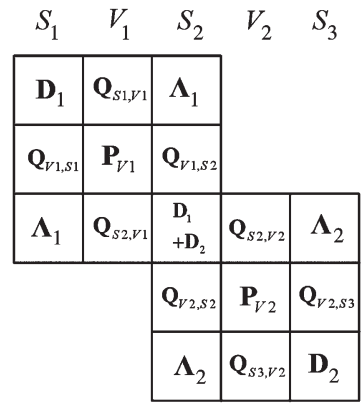

(a)

(b)

Fig. 2. (a) Prism-element-based discretization and unknown ordering scheme. (b) Three-dimensional layered system matrix.

and $\Delta t$ represents the time step. Obviously, $u^{n+1}$ (the field value at the $(n+1)$ th time step) can be solved in a time marching fashion from the solution of $u$ at previous time steps.

Matrix $\tilde{\mathbf{T}}$ can be very large for realistic on-chip and package problems, which constitutes a computational challenge. This challenge can be overcome by the time-domain OrFE-RR method described in the following section.

\section{B. OrFE-RR Method}

We discretize the computational domain into layers of prism elements as shown in Fig. 2(a). Conductors are also discretized in order to capture the internal fields accurately. In each element, the electric field is expanded into orthogonal vector basis functions described in Section II. The unknowns are ordered layer by layer. In each layer, the unknowns are divided into surface and volume ones. As shown in Fig. 2(a), the unknowns associated with the horizontal edges are surface unknowns, and the unknowns associated with the vertical edges are volume unknowns. The unknowns are then ordered from $S_{1}$ to $V_{1}$ to $S_{2}$ to $V_{2}$ and so on, resulting in a 3-D layered system matrix shown in Fig. 2(b). In this matrix, all the $\mathbf{D}_{l}$ and $\Lambda_{l}(l=1,2, \ldots, L)$ matrices are diagonal due to the orthogonality of the vector basis functions as shown in (8). Since the surface vector bases $\mathbf{N}_{i}(i=1-6,10-15)$ are perpendicular to volume vector bases $\mathbf{N}_{i}(i=7-9)$, as can be seen from Fig. 1 , and $\tilde{\mathbf{T}}$ in (15) solely comprises matrices formed by the inner product of $\mathbf{N}_{i}$ and $\mathbf{N}_{j}$, all the $\mathbf{Q}$ matrices in Fig. 2(b) vanish.

1) Reduction From a 3-D Layered System to a SingleLayer One That Is Diagonal in Negligible Computational Cost: Since all the $\mathbf{Q}$ matrices vanish, the 3-D layered system is naturally decomposed into a 2-D layered system formed by surface unknowns only, as shown in Fig. 3(a), and $L$ decoupled matrices formed by volume unknowns in each layer only, as shown in Fig. 3(b). This step certainly is free of any CPU and memory cost. As a result, the matrix equation (14) is naturally decomposed into

$$
\begin{aligned}
\mathbf{P}_{S} x_{S} & =b_{S} \\
\mathbf{P}_{V l} x_{V l} & =b_{V l}, \quad l=1,2, \ldots, L
\end{aligned}
$$

in which $\mathbf{P}_{S}$ is the 2-D layered system shown in Fig. 3(a), $\mathbf{P}_{V l}$ is the volume-unknown-based system in layer $l$ shown in Fig. 3(b), and $x_{S}$ and $x_{V}$ represent the surface and volume unknowns, respectively. 


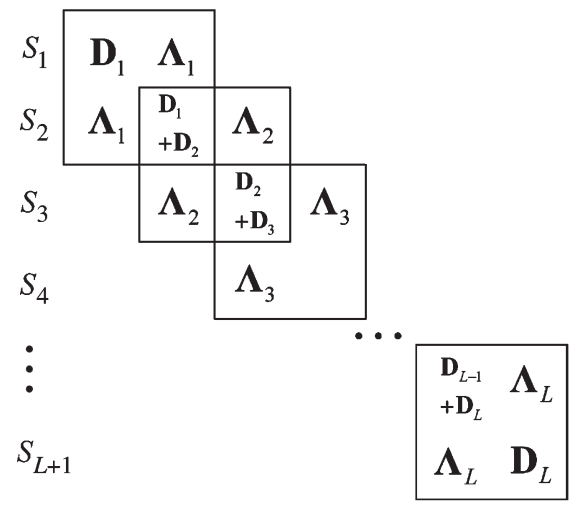

(a)

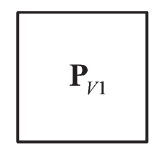

$V_{1}$

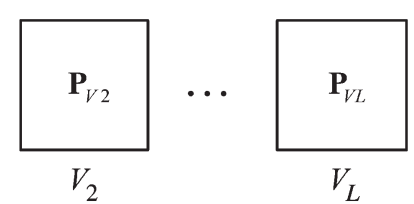

(b)
Fig. 3. (a) Two-dimensional layered system matrix formed by surface unknowns only. (b) $L$ decoupled matrices formed by volume unknowns in each layer only.

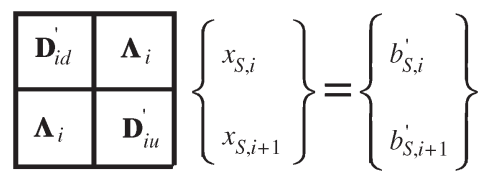

Fig. 4. Reduced single-layer system.

The 2-D layered system is then reduced to a single-layer system matrix shown in Fig. 4, in which matrix $\mathbf{D}_{i d}^{\prime}$ carries the contribution of all the layers above layer $i$ to layer $i$, while matrix $\mathbf{D}_{i u}^{\prime}$ carries the contribution of all the layers below layer $i$ to layer $i$. These two matrices can be obtained recursively from

$$
\begin{aligned}
\mathbf{D}_{2}^{\prime}= & \mathbf{D}_{1}+\mathbf{D}_{2}-\boldsymbol{\Lambda}_{1} \mathbf{D}_{1}^{-1} \boldsymbol{\Lambda}_{1} \\
\mathbf{D}_{3}^{\prime}= & \mathbf{D}_{2}+\mathbf{D}_{3}-\boldsymbol{\Lambda}_{2} \mathbf{D}_{2}^{\prime-1} \boldsymbol{\Lambda}_{2} \\
& \vdots \\
\mathbf{D}_{i d}^{\prime}= & \mathbf{D}_{i-1}+\mathbf{D}_{i}-\boldsymbol{\Lambda}_{i-1} \mathbf{D}_{i-1}^{\prime-1} \boldsymbol{\Lambda}_{i-1} \\
\mathbf{D}_{L-1}^{\prime}= & \mathbf{D}_{L-1}+\mathbf{D}_{L}-\boldsymbol{\Lambda}_{L} \mathbf{D}_{L}^{-1} \boldsymbol{\Lambda}_{L} \\
\mathbf{D}_{L-2}^{\prime}= & \mathbf{D}_{L-2}+\mathbf{D}_{L-1}-\boldsymbol{\Lambda}_{L-1} \mathbf{D}_{L-1}^{\prime-1} \boldsymbol{\Lambda}_{L-1} \\
& \vdots \\
\mathbf{D}_{i u}^{\prime} & =\mathbf{D}_{i}+\mathbf{D}_{i+1}-\boldsymbol{\Lambda}_{i+1} \mathbf{D}_{i+1}^{\prime-1} \boldsymbol{\Lambda}_{i+1} .
\end{aligned}
$$

Since all the $\mathbf{D}_{l}$ and $\Lambda_{l}(l=1,2, \ldots, L)$ are diagonal, $\mathbf{D}_{i d}^{\prime}$ and $\mathbf{D}_{i u}^{\prime}$ are obtained in negligible cost. In addition, $\mathbf{D}_{i d}^{\prime}$ and $\mathbf{D}_{i u}^{\prime}$ are diagonal.

The right-hand side in the reduced single-layer system shown in Fig. 4 can also be obtained recursively from the right-hand side of the original system [11], [12]. Again, in this process, there is no need to perform matrix factorization and matrixvector multiplication since $\mathbf{D}_{l}$ and $\Lambda_{l}$ are diagonal.

The single-layer system can be further reduced to a singleinterface system shown in Fig. 5, where

$$
\begin{aligned}
\mathbf{D}_{i d}^{\prime \prime} & =\mathbf{D}_{i, d}^{\prime}-\boldsymbol{\Lambda}_{i} \mathbf{D}_{i u}^{\prime-1} \boldsymbol{\Lambda}_{i} \\
b_{S, i}^{\prime \prime} & =b_{S, i}^{\prime}-\boldsymbol{\Lambda}_{i} \mathbf{D}_{i u}^{\prime-1} b_{S, i+1}^{\prime} .
\end{aligned}
$$

$$
\mathbf{D}_{i}^{\prime \prime}\left\{x_{S, i}\right\}=\left\{b_{S, i}^{\prime}\right\}
$$

Fig. 5. Reduced single-interface system.

Again, the operations in (19) and (20) only involve diagonal matrices, the cost of which is negligible. In addition, the reduced single-interface system is also diagonal and, hence, can be solved readily.

2) Recovery of Other Unknowns in Linear Complexity: Once the unknowns in the reduced system shown in Fig. 5 is known, the surface unknowns in other layers can be recovered from

$$
\begin{array}{rlrl}
x_{S, l} & =\mathbf{D}_{l}^{\prime-1}\left[b_{S, l}^{\prime}-\boldsymbol{\Lambda}_{l} x_{S, l+1}\right], & & l=i-1, i-2, \ldots, 1 \\
x_{S, l+1} & =\mathbf{D}_{l}^{\prime-1}\left[b_{S, l+1}^{\prime}-\boldsymbol{\Lambda}_{l} x_{S, l}\right], & & l=i+1, \ldots, L-1 \\
x_{S, l+1} & =\mathbf{D}_{l}^{-1}\left[b_{S, l+1}-\boldsymbol{\Lambda}_{l} x_{S, l}\right], & l=L .
\end{array}
$$

Clearly, the aforementioned operations have linear complexity due to diagonal matrices $\mathbf{D}_{l}$ and $\Lambda_{l}$.

The volume unknowns are recovered layer by layer from

$$
x_{V l}=\mathbf{P}_{V l}^{-1} b_{V l}, \quad l=1,2, \ldots, L
$$

in which $\mathbf{P}_{V l}$ is assembled from

$$
\begin{aligned}
\mathbf{P}_{V l, i j}^{e}=h_{l}\left(\mu_{0} \varepsilon_{l}^{e}+0.5 \Delta t \mu_{0} \sigma_{l}^{e}\right)\left\langle\xi_{i}, \xi_{j}\right\rangle_{\Omega^{e}}, & \\
& \quad l=1,2, \ldots, L .
\end{aligned}
$$

$\mathbf{P}_{V l}$ is a sparse matrix, the dimension of which is approximately half of the number of unknowns in a single layer. If the size of the single-layer system is small, $\mathbf{P}_{V l}$ can be readily solved. However, if the size of the single-layer system is large, solving $\mathbf{P}_{V l}$ can be a numerical challenge. In the following, we present an algorithm of solving $\mathbf{P}_{V l}$ in linear complexity.

Without loss of generality, assuming that the volume unknowns are assigned along the $z$-direction, Fig. 6(a) shows an $x-y$ cross-sectional view of these volume unknowns, in which each dot denotes a volume unknown. If the unknowns are ordered from line 1 to line 2 to line 3 , and to line $N_{x}$, a $\mathbf{P}_{V l}$ matrix having the pattern shown in Fig. 6(b) will be generated. It is a block tridiagonal matrix. More importantly, each diagonal block is a tridiagonal matrix. The off-diagonal blocks are very sparse. For example, for the mesh shown in Fig. 6(a), each row has only two nonzero elements (or even less); one is the diagonal one, and the other is the super- or subdiagonal one.

To fully explore the structure of $\mathbf{P}_{V l}$ to expedite its solution, we split $\mathbf{P}_{V l}$ as the following:

$$
\mathbf{P}_{V l}=\mathbf{P}-\mathbf{L}-\mathbf{U}
$$

where $\mathbf{P}$ is the block diagonal matrix, $-\mathbf{L}$ is the strict lower triangular part, and $-\mathbf{U}$ is the strict upper triangular part. The matrix patterns of $\mathbf{P}, \mathbf{L}$, and $\mathbf{U}$ are shown in Fig. 6(c). $\mathbf{L}$ and $\mathbf{U}$ are sparse matrices. For the mesh shown in Fig. 6(a), $\mathbf{L}$ and $\mathbf{U}$ are bidiagonal matrices.

The matrix $\mathbf{P}$ serves as an effective preconditioner of $\mathbf{P}_{V l}$. It was shown by numerical experiments that, with $\mathbf{P}$, the iterative process of solving (22) converges in a few iterations for the realistic on-chip and package examples we have tested. 


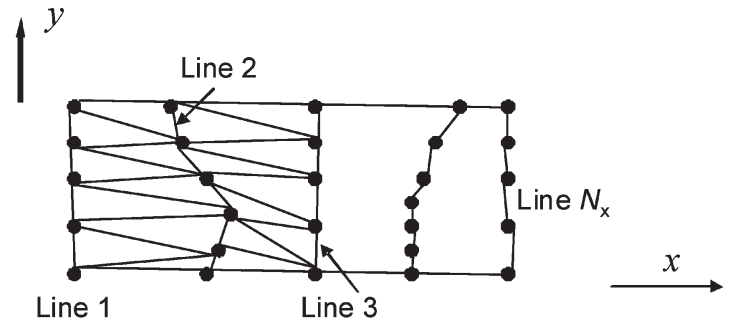

(a)

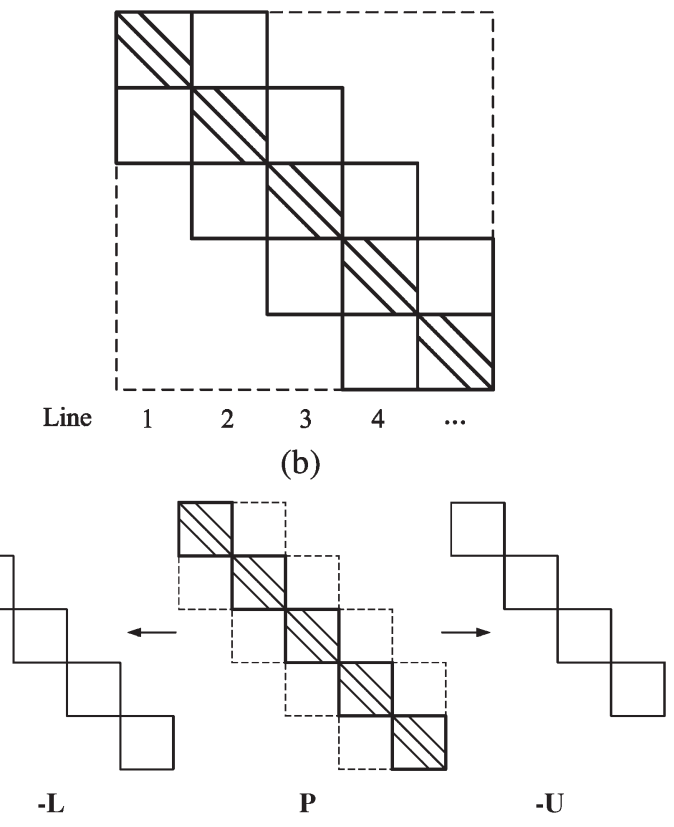

(c)

Fig. 6. (a) Top view of the volume unknowns in a single layer. (b) Matrix pattern of $\mathbf{P}_{V l}$. (c) Splitting of $\mathbf{P}_{V l}$

By using $\mathbf{P}$ as the preconditioner, (22) is solved iteratively as

$$
\mathbf{P} x^{(k+1)}=(\mathbf{L}+\mathbf{U}) x^{(k)}+b_{V l}, \quad k \geq 0
$$

with $k$ being the iteration number. When (25) converges, $x^{(k+1)}=x^{(k)}$, and hence, $(\mathbf{P}-\mathbf{L}-\mathbf{U}) x^{(k+1)}=b_{V l}$. From (24), it can be seen that $x^{(k+1)}$ is the solution of (22). In the Appendix, we give a proof to show that the iteration in (25) converges for any right-hand side $b_{V l}$ and any initial vector $x^{(0)}$.

Since $\mathbf{P}_{V l}$ is a block tridiagonal matrix, (25) can be simplified to

$\mathbf{P}_{i i} x_{i}^{(k+1)}=\mathbf{L}_{i, i-1} x_{i-1}^{(k)}+\mathbf{U}_{i, i+1} x_{i+1}^{(k)}+d_{i}, \quad 1 \leq i \leq N_{x}$

in which $i$ denotes the line index and $\mathbf{P}_{i i}$ denotes the $i$ th diagonal block of $\mathbf{P}$, which is formed by volume unknowns on line $i$. Since $\mathbf{P}_{i i}$ is a tridiagonal matrix, as shown in [15], its inverse can be stored efficiently in two sequences $\left\{u_{j}\right\}$ and $\left\{v_{j}\right\}, j=1,2, \ldots, m$, with $m$ being the dimension of $\mathbf{P}_{i i}$. Meanwhile, $\mathbf{P}_{i i}^{-1} b$ can be performed in linear complexity [15].

3) Performance Analysis: The numerical procedure of the proposed OrFE-RR method can be summarized as follows.

Step 1) Reduce the original 3-D layered system matrix to a single-interface system (shown in Fig. 5) that is diagonal.
Step 2) Precalculate the inverse of $\mathbf{P}_{i i}$ in (26).

Beginning of the time marching.

Step 3) Solve the reduced system shown in Fig. 5 and obtain the solution of a single surface.

Step 4) Recover solutions of surface unknowns in all other layers using (21) and recover solutions of volume unknowns in all layers using (22).

Step 5) Construct the new right-hand side of (14) for the next time step. Go back to Step 3).

End of the time marching.

The computational cost of each step is analyzed as follows.

Step 1) The cost is negligible due to orthogonal prism vector basis functions that render $\mathbf{D}_{l}$ and $\Lambda_{l}(l=$ $1,2, \ldots, L)$ diagonal.

Step 2) Both memory and CPU costs are linear because $\mathbf{P}_{i i}$ is a tridiagonal matrix. Assuming that the number of unknowns along a single line in Fig. 6(a) is $m$, the memory and CPU cost of calculating and storing $\mathbf{P}_{i i}^{-1}$ is $O(m)$.

Step 3) The cost is negligible since the reduced system is diagonal.

Step 4) Both memory and CPU costs are linear.

Step 5) The cost is a few sparse matrix-vector multiplications, which has a linear complexity.

Overall, in the proposed OrFE-RR method, the reduction from a 3-D layered system to a single-layer one costs negligible computational resources; the reduced system is diagonal, and hence, its solution time is minimized; the complexity of recovery scales linearly with the number of unknowns to be recovered. The method applies to any arbitrarily shaped multilayer structure embedded in inhomogeneous media. The time step permitted by this method is the same as that permitted by the conventional TDFEM schemes.

\section{Numerical RESUlts}

To validate the accuracy of the proposed time-domain OrFERR method, we first simulated a parallel-plate waveguide, the analytical solution of which is known. According to typical geometrical dimensions of on-chip problems, the waveguide width (along the $y$-direction) was set as $1 \mu \mathrm{m}$, the waveguide height (along the $x$-direction) was set as $0.1 \mu \mathrm{m}$, and the waveguide length (along the $z$-direction) was set as $3.5 \mu \mathrm{m}$. The structure was divided into 35 layers along the length. An incident transverse electric magnetic plane wave was launched at the near end of the waveguide (at $z=0$ ). An exact absorbing boundary condition is placed at both the near and far ends of the waveguide to absorb the outgoing wave. The excitation was a time derivative of a Gaussian pulse $\hat{E}^{\text {inc }}(t)=$ $\hat{x} 2 t \exp \left(-t^{2} / \tau^{2}\right)$, in which $\tau=3.0 \times 10^{-13} \mathrm{~s}$. The structure was discretized into triangular prism elements, resulting in 8067 unknowns. The time step was chosen as $0.7 \times 10^{-16} \mathrm{~s}$. In total, 20000 time steps were simulated. The electric field at $1.7 \mu \mathrm{m}$ was sampled. As can be seen from Fig. 7(a), the result obtained from the proposed OrFE-RR method shows an excellent agreement with the analytical solution. Fig. 7(b) and (c) shows the electric fields sampled at $z=0.1,1.7$, and $3.5 \mu \mathrm{m}$. 


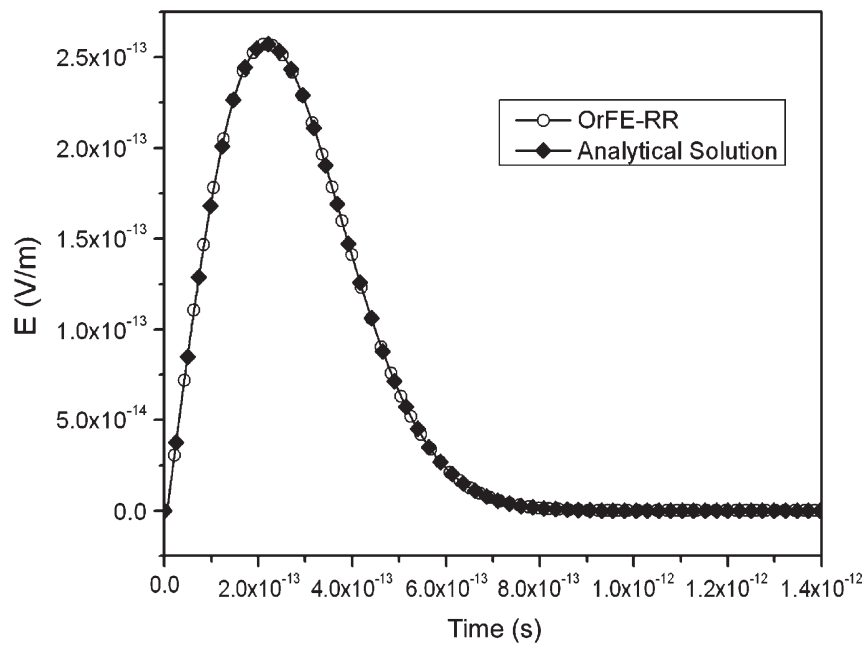

(a)

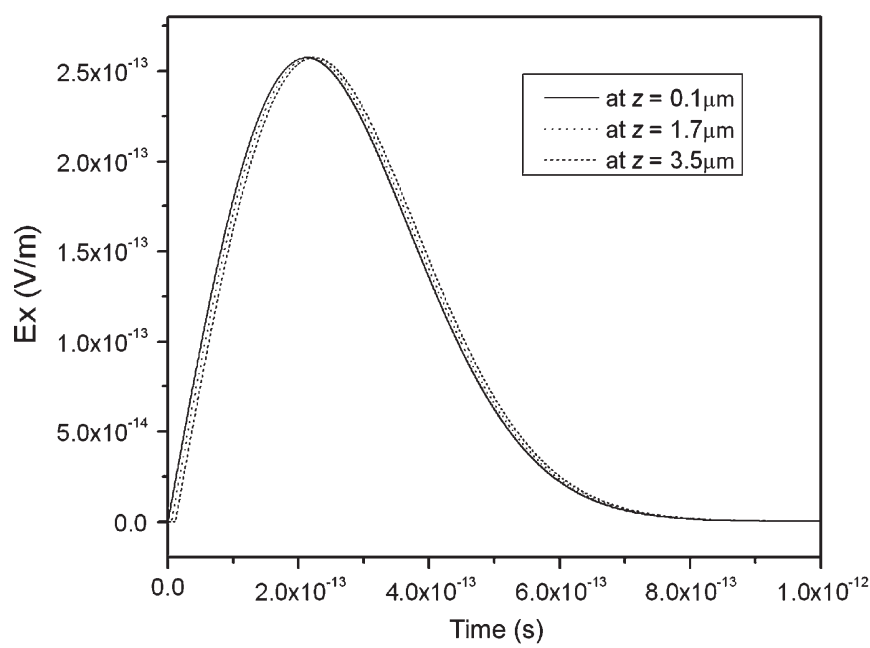

(b)

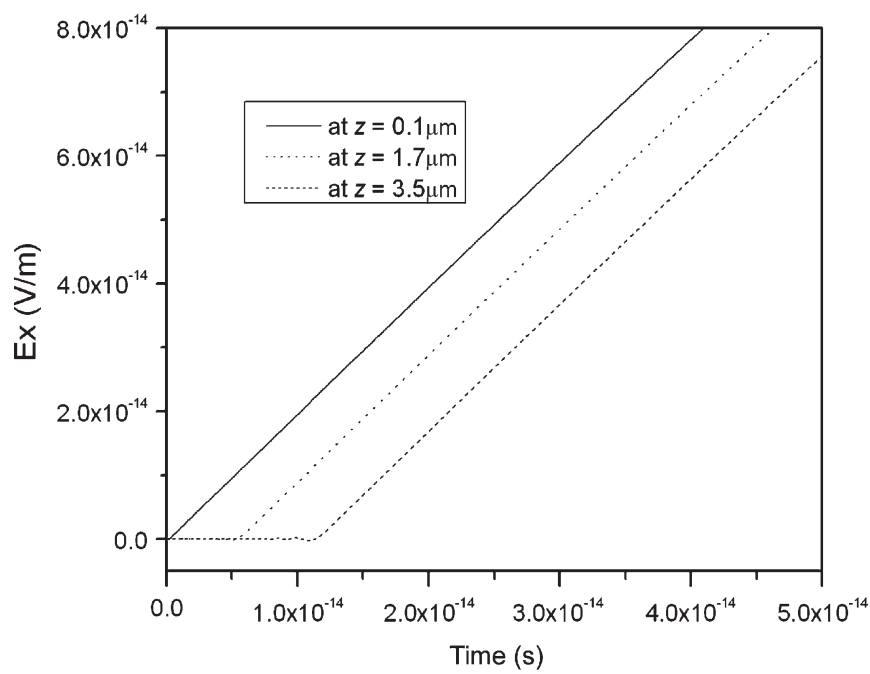

(c)

Fig. 7. Simulation of a parallel-plate waveguide. (a) Electric field at $1.7 \mu \mathrm{m}$. (b) Electric fields at $z=0.1,1.7$, and $3.5 \mu \mathrm{m}$ simulated by the proposed method and those obtained from a conventional TDFEM solver. (c) Magnified plot showing the time delay.

The waveforms and time delay obtained by the proposed method were compared with those simulated by a conventional TDFEM solver. The results are on top of each other.

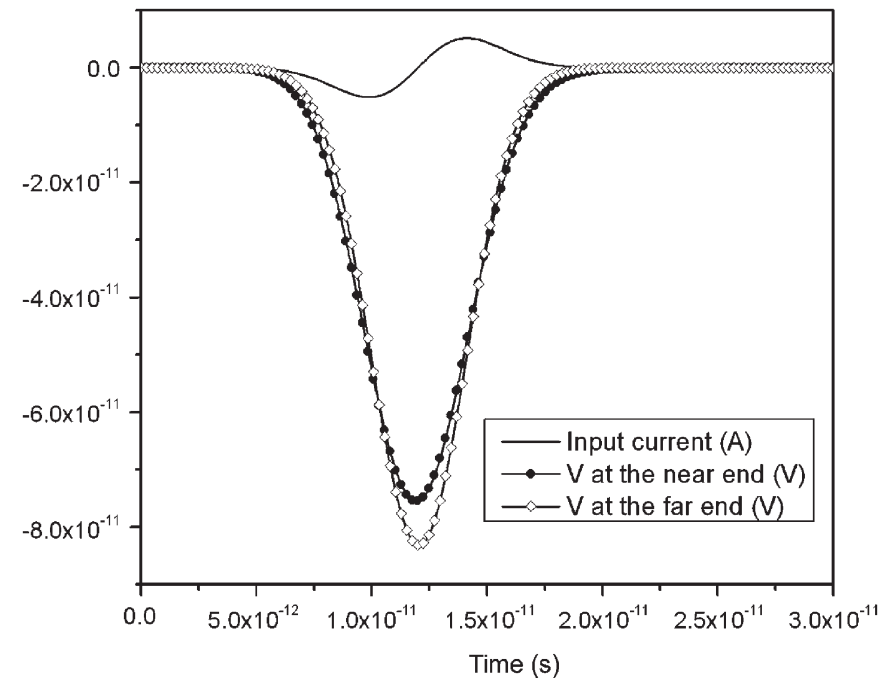

Fig. 8. Time-domain waveform of the input current and voltages sampled at the near and far ends of a test-chip interconnect structure of $100-\mu \mathrm{m}$ length.

With the accuracy validated, a test-chip interconnect structure with a length of $100 \mu \mathrm{m}$ was simulated. The test-chip interconnect structure was fabricated using conventional silicon processing technology [14]. It comprised three metal layers and thirteen inhomogeneous dielectric stacks. The structure was divided into ten layers along its length, resulting in 30973 unknowns. A current source was placed at the near end. The far end was left open. The time step used in this simulation is $1.2 \times 10^{-16} \mathrm{~s}$. Fig. 8 shows the time-domain waveforms of the voltages sampled at the near and far ends of one wire in the interconnect structure. Fig. 9 shows the frequency-domain S-parameters obtained from the proposed method in comparison with the measured data. Excellent agreement is observed.

Next, a test-chip interconnect structure of 2000- $\mu \mathrm{m}$ length was simulated. The structure was discretized into 127093 unknowns. The time step was chosen as $1.2 \times 10^{-16} \mathrm{~s}$. The input current and the voltage waveforms sampled at the near and far ends of one wire in the interconnect structure are shown in Fig. 10, which demonstrates a clear inductance effect. The simulated S-parameters in comparison with the measured data are shown in Fig. 11. Again, excellent agreement is observed.

After demonstrating the accuracy of the proposed method, we tested its performance in comparison with the conventional TDFEM that employs a state-of-the-art multifrontal-based sparse matrix solver, UMFPACK [24]. The on-chip interconnect structure of $2000-\mu \mathrm{m}$ length was chosen as the testing example. The meshing on the $x y$ plane was fixed while the number of layers along the length ( $z$-direction) was varied to generate approximately 20000 to 30 million unknowns. Fig. 12 shows the CPU and memory cost as a function of the number of unknowns. Obviously, the proposed method outperforms the conventional method in both CPU time and memory consumption. More importantly, the proposed method scales favorably with the number of unknowns. Since the cost of the reduction in the proposed method is negligible, the computation is solely spent on recovering $N$ unknowns of interest, which clearly shows a linear complexity as can be seen from Fig. 12. To be specific, Fig. 12(a) and (b) shows the CPU cost and memory 

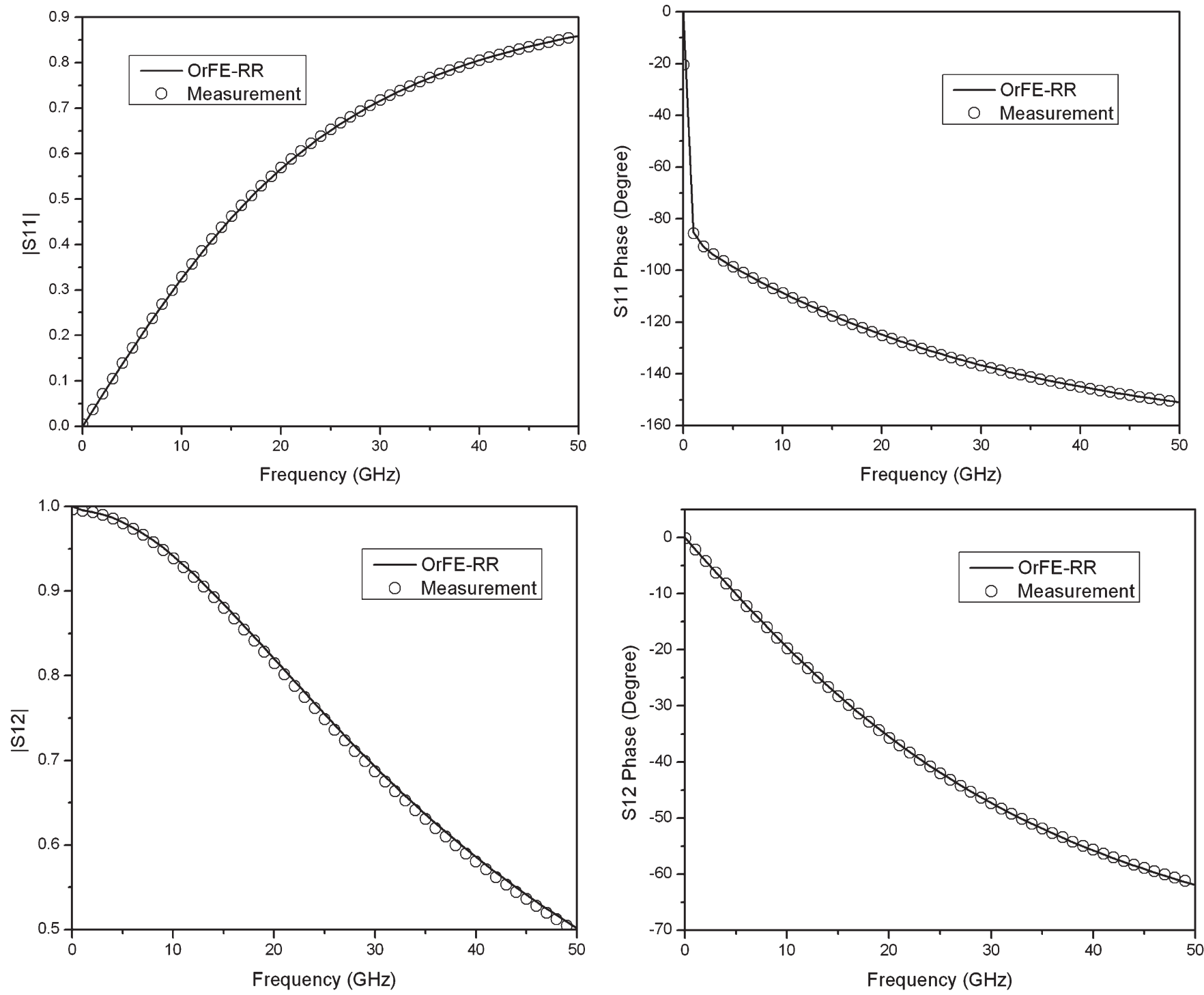

Fig. 9. S-parameters of a 100- $\mu$ m-long test-chip interconnect simulated by the proposed OrFE-RR method.

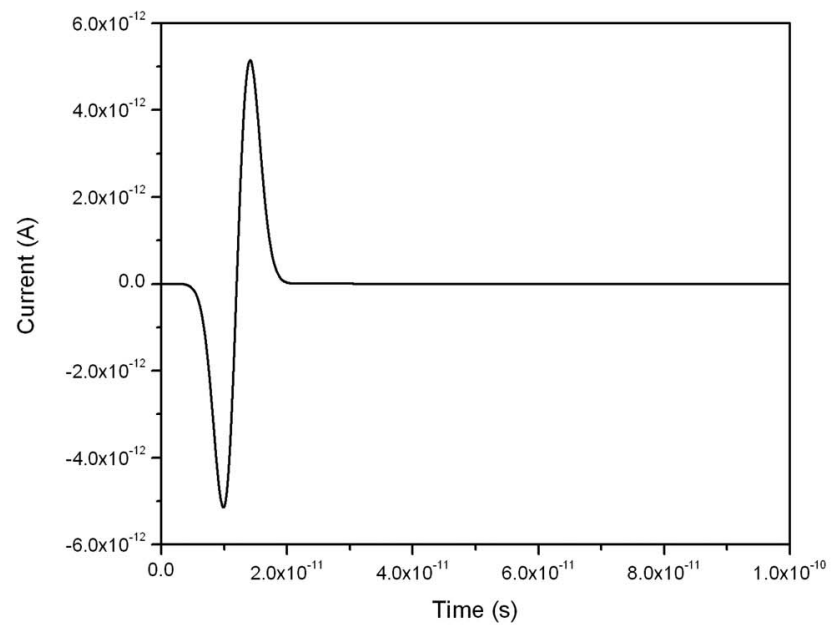

(a)

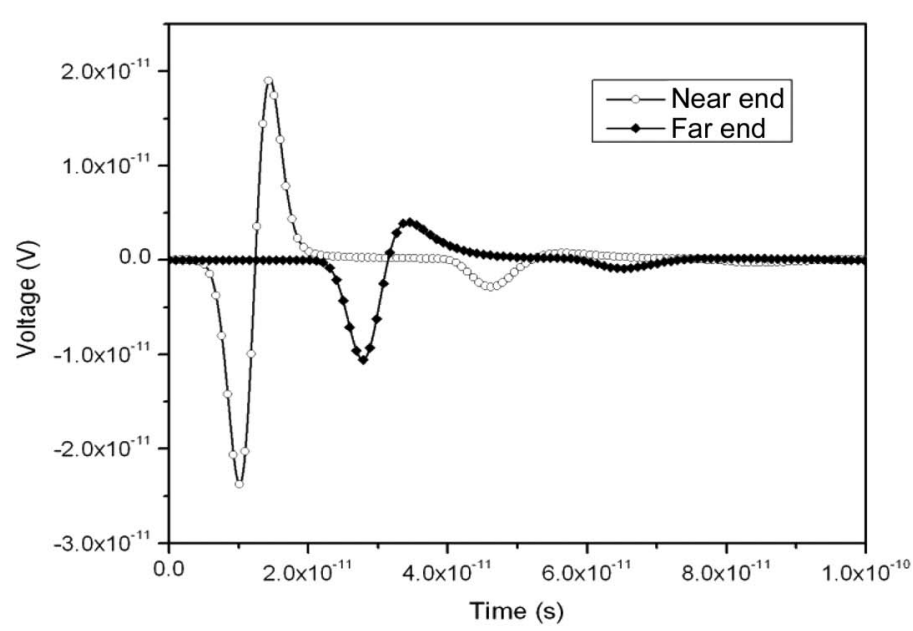

(b)

Fig. 10. Simulation of a test-chip interconnect of 2000- $\mu$ m length. (a) Input current. (b) Voltages sampled at the near and far ends of one wire in the interconnect structure. 


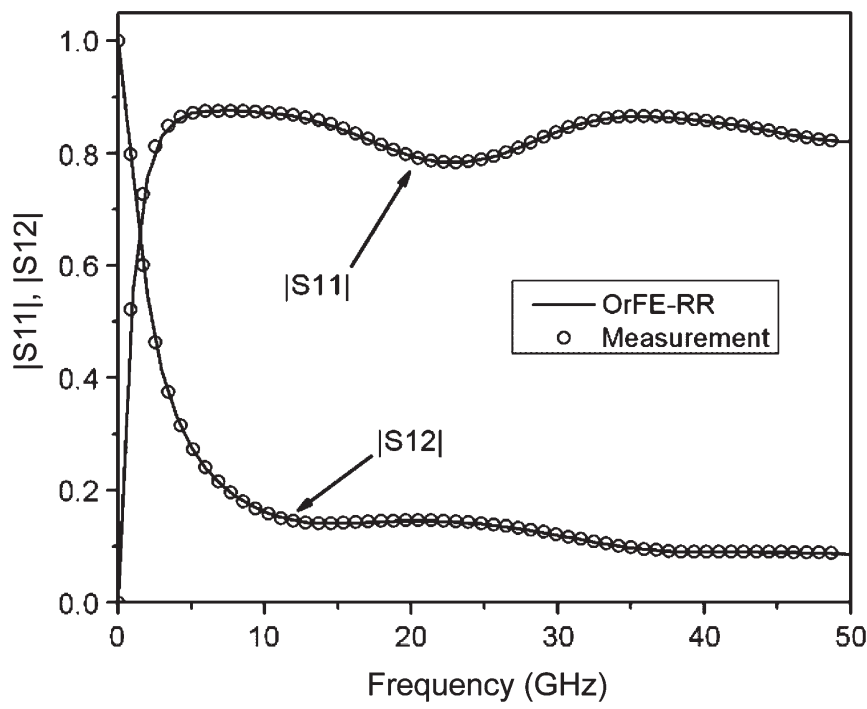

Fig. 11. S-parameters of a 2000- $\mu$ m-long test-chip interconnect simulated by the proposed OrFE-RR method.

consumption in factorization, respectively. The conventional method has to factorize the entire system matrix $\tilde{\mathbf{T}}$ in (15), whereas the proposed method only needs to invert $\mathbf{P}_{i i}$ in (26), which has a 1-D size and is tridiagonal. Fig. 12(c) shows the matrix solving time. The conventional method has to perform backward-forward substitutions of a matrix that has a 3-D size, whereas the proposed method deals with the multiplication of $\mathbf{P}_{i i}^{-1}$ with a vector, which has a 1-D size, and, moreover, can be done in linear complexity.

In Fig. 12, the complexity curve of the conventional TDFEM solver was not drawn to the end because, when the number of layers exceeded 100, yielding more than $1.5 \times 10^{5}$ unknowns, the conventional solver based on [24] failed to factorize the system matrix. In addition, from Fig. 12, it can be seen that, even if the number of unknowns is in the range that the conventional solver is applicable, the complexity is not linear.

The convergence performance of the iterative solution of (22) is shown in Fig. 13. Clearly, the proposed method only requires a few iterations to achieve good accuracy when recovering volume unknowns.

Next, we tested the capability of the OrFE-RR method in modeling irregular geometry. A real package structure (provided by Intel Corporation) was simulated. The structure involved a bottom ground plane with via holes, a center via layer consisting of both power and ground vias, and a top power plane with via holes, occupying an area of $2500 \times 2500 \mu \mathrm{m}$. A bottom negative supply voltage (VSS) via was excited by a current source. An unstructured mesh was used to capture the irregular geometry of the vias and holes. The voltage maps of the positive supply voltage (VCC) and VSS package planes were shown in Fig. 14(a) and (b). Although it was the VSS via that was excited, the coupling to the VCC plane can be clearly seen.

We then simulated a $2500 \times 2500 \mu \mathrm{m}$ combined diepackage power delivery system. An illustration of this example is shown in Fig. 15. The package structure was kept the same. Three on-chip layers were added. VCC and VSS vias traversed via holes and contacted package planes. The on-chip power rails were connected through on-chip vias. A current source

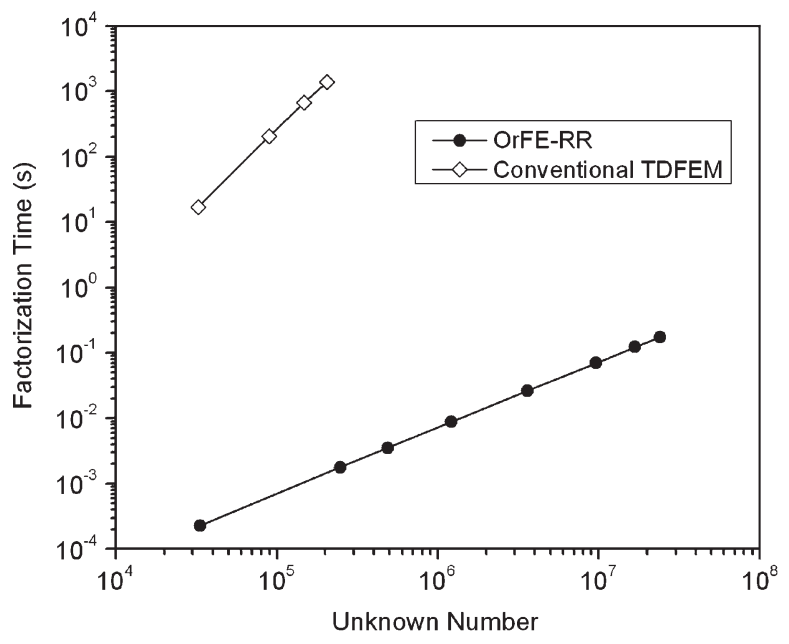

(a)

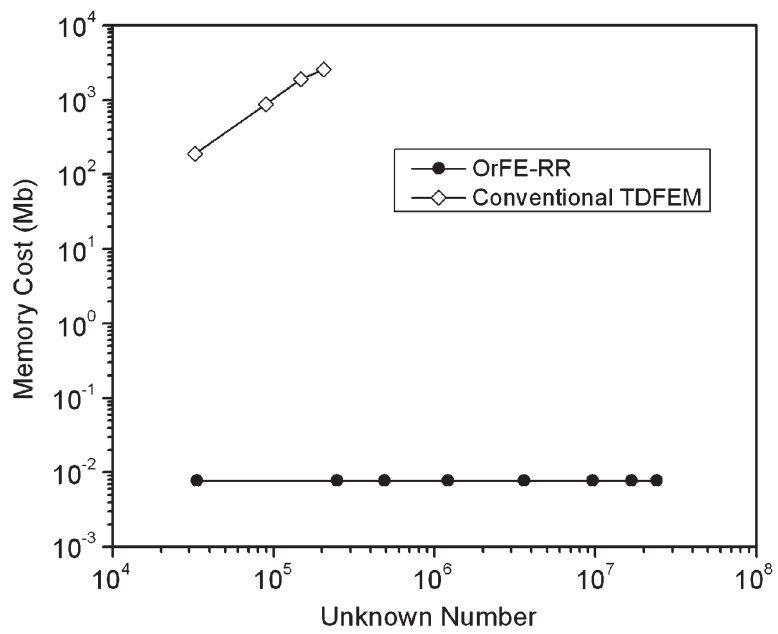

(b)

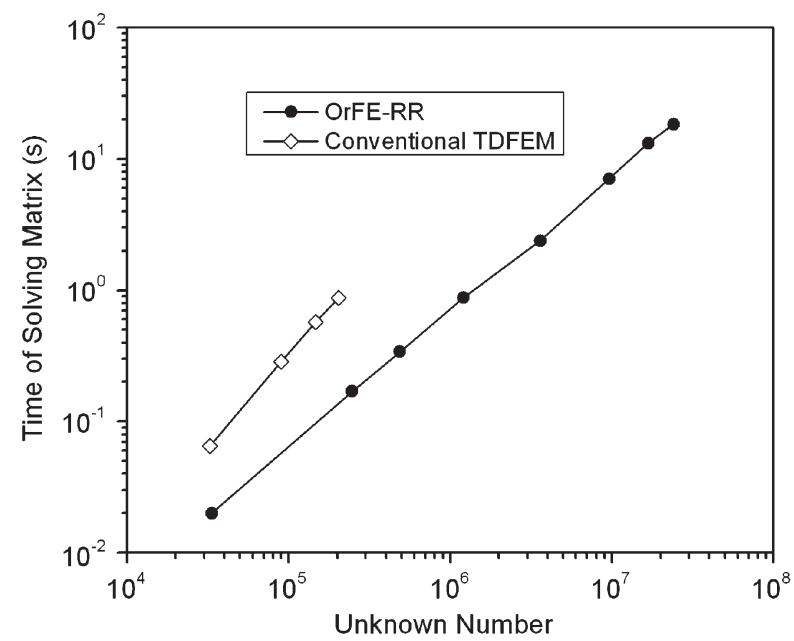

(c)

Fig. 12. (a) Time complexity of the matrix factorization. (b) Memory complexity of the matrix factorization. (c) CPU cost at each time step as a function of $N$.

was launched at a VSS via from the silicon. In Fig. 16, the dynamic voltage maps of the package and on-chip layers were plotted at the 6000th time step. In this simulation, a time step of $2.5 \times 10^{-15} \mathrm{~s}$ was used. The CPU cost at each time step was $0.51846 \mathrm{~s}$. 


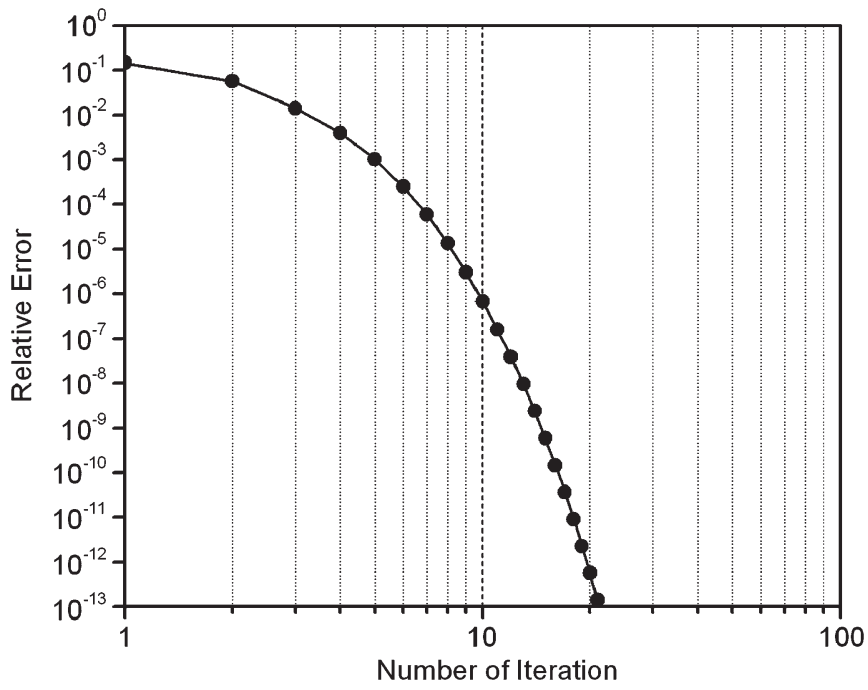

Fig. 13. Relative error versus the number of iterations in solving $\mathbf{P}_{V l}$.

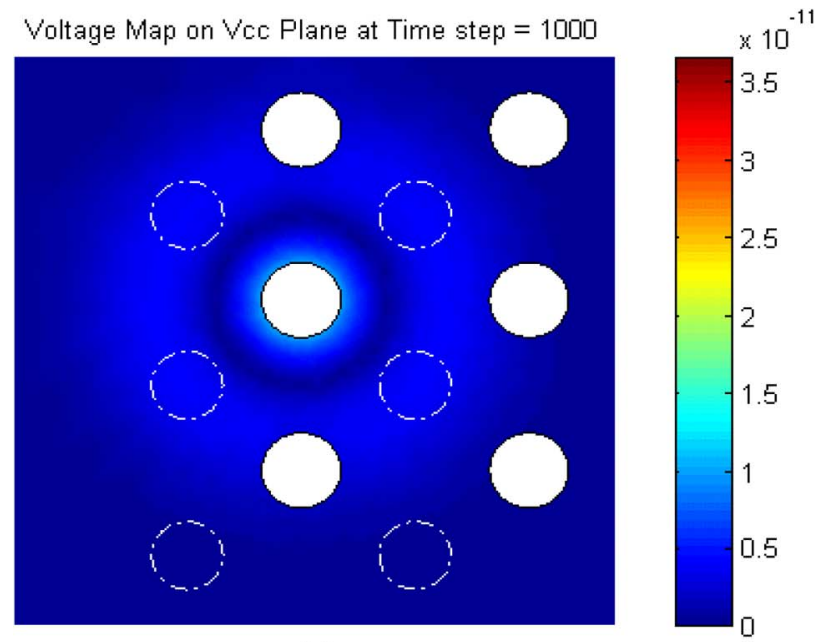

(a)

Voltage Map on Vss Plane at Time step = 1000

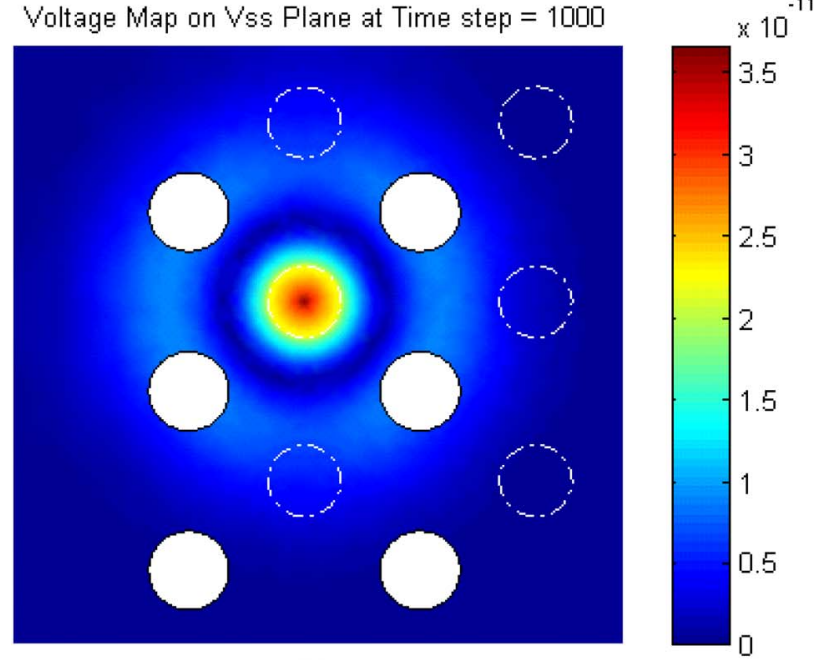

(b)

Fig. 14. Simulation of a package power delivery structure. (a) Voltage map of a VCC plane sampled at the 1000th time step. (b) Voltage map of a VSS plane sampled at the 1000th time step.

\section{CONCLUSION}

In this paper, a set of orthogonal prism vector basis functions have been developed. These bases are complete in representing fields. In addition, they enforce the tangential continuity of the electric field and the normal continuity of the electric flux across material interfaces. Based on this set of bases, a timedomain OrFE-RR method was developed to solve large-scale IC and package problems. This method is capable of reducing a 3-D layered system matrix to a single-layer system matrix with negligible cost, irrespective of the original problem size. In addition, the reduced single-layer system matrix is diagonal and, hence, can be solved readily. From the solution of the reduced system, the solutions of other unknowns are recovered in linear complexity. The method applies to any arbitrarily shaped multilayer structure involving inhomogeneous materials. It entails no theoretical approximation. Numerical and experimental results have demonstrated its superior performance in simulating onchip, package, and combined die-package problems.

\section{APPENDIX}

Define a sequence of iterations of the form

$$
x^{(k+1)}=\mathbf{G} x^{(k)}+f
$$

in which $\mathbf{G}$ is a square matrix. It is known that, if the spectral radius of $\mathbf{G}$, denoted by $\rho(\mathbf{G})$, is less than 1 , then $\mathbf{I}-\mathbf{G}$ is nonsingular, and the iteration in (A.1) converges for any $f$ and $x^{(0)}$ [25].

From (25), it can be seen that $\mathbf{G}$ in our iteration scheme is

$$
\mathbf{G}=\mathbf{P}^{-1}(\mathbf{L}+\mathbf{U}) .
$$

Denoting the eigenvalues of $\mathbf{G}$ by $\lambda$, we have

$$
\mathbf{G} v=\lambda v
$$

in which $v$ denotes the eigenvector. From (A.2) and (A.3), we obtain

$$
(\mathbf{L}+\mathbf{U}) v=\lambda \mathbf{P} v .
$$

Taking a norm on both sides of (A.4), we obtain

$$
|\lambda|=\frac{\|(\mathbf{L}+\mathbf{U}) v\|}{\|\mathbf{P} v\|}
$$

in which $\|(\cdot)\|$ denotes the norm of $(\cdot)$. Without loss of generality, the 1-norm of a vector is used, which is

$$
\|g\|_{1}=\sum_{i=1}^{m}\left|g_{i}\right|
$$

where $m$ is the length of vector $g$. If $\|(\mathbf{L}+\mathbf{U}) v\|$ is less than $\|\mathbf{P} v\|$, then the modulus of $\lambda$ is less than 1 , then $\rho(\mathbf{G})<1$, and then the convergence of (25) is proved.

Matrices $\mathbf{L}$ and $\mathbf{U}$ are the off-diagonal blocks of $\mathbf{P}_{V l}$, and $\mathbf{P}$ is the diagonal block. From (23), the matrix elements of $\mathbf{P}_{V l}$ can be written as

$$
\mathbf{P}_{V l, m n}^{e}=h_{l}\left(\mu_{0} \varepsilon_{l}^{e}+0.5 \Delta t \mu_{0} \sigma_{l}^{e}\right)\left\langle\xi_{m}, \xi_{n}\right\rangle_{\Omega^{e}}
$$




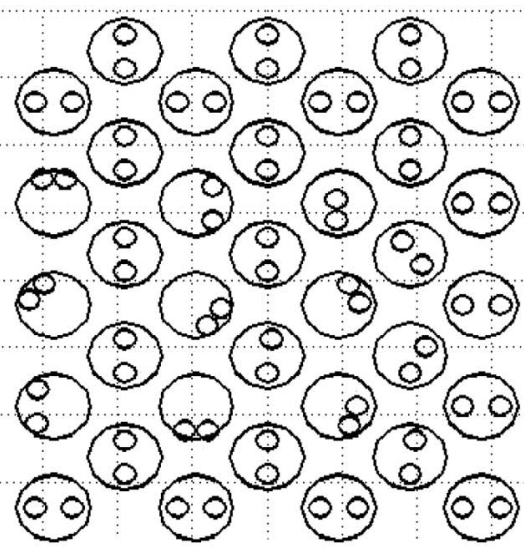

(a)

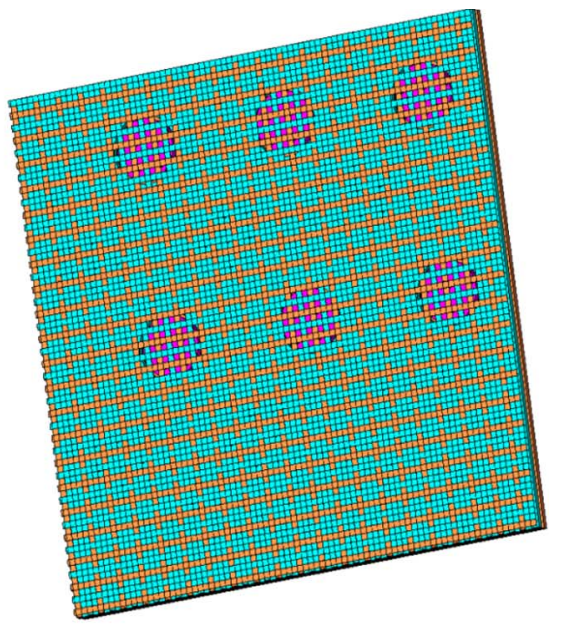

(b)

Fig. 15. Illustration of the die-package power delivery example. (a) Top view from the package side (Big circles are holes; small circles are sampled via contacts). (b) Bottom view from the on-chip side.

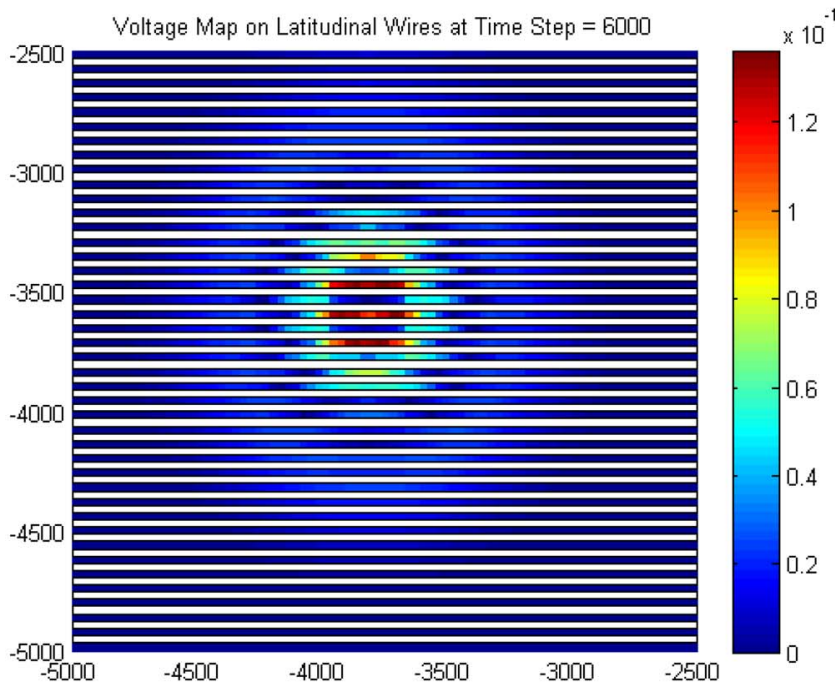

(a)

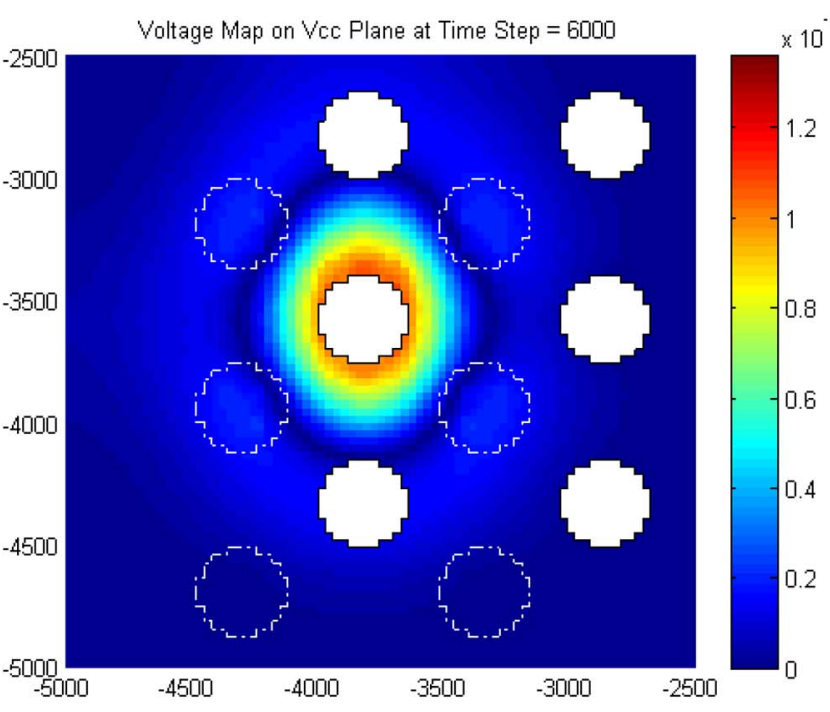

(c)

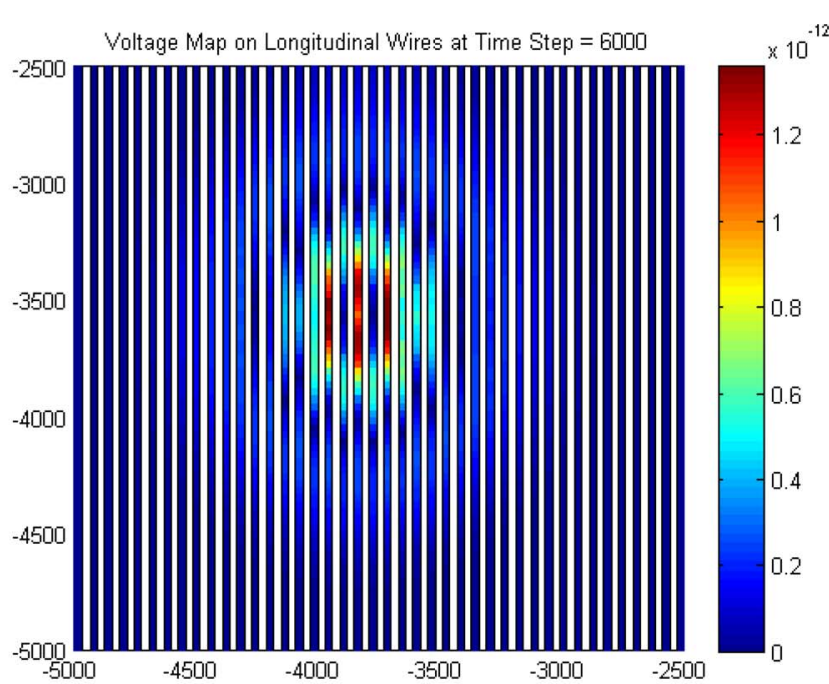

(b)

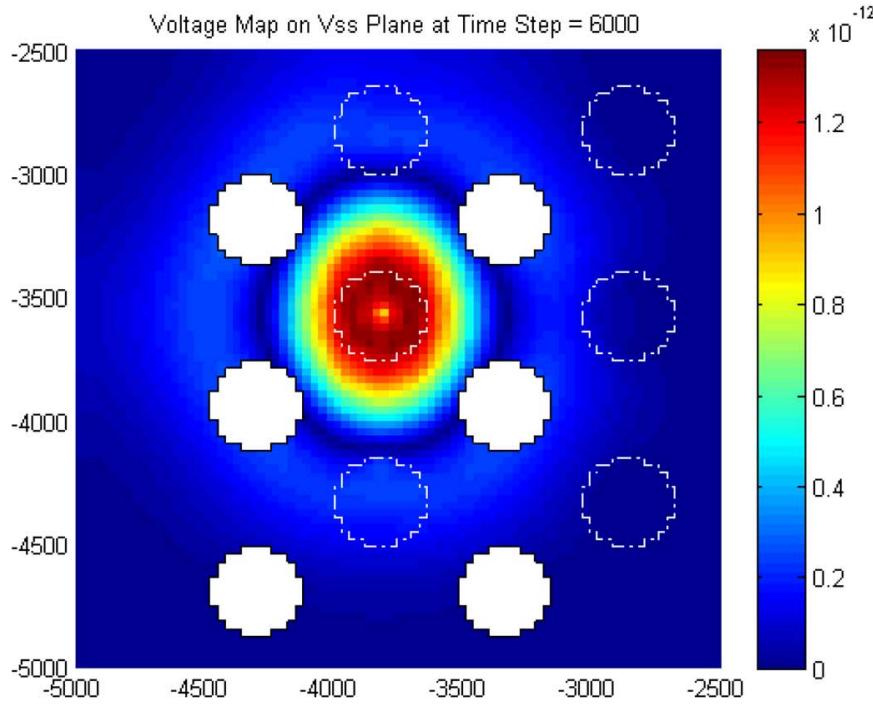

(d)

Fig. 16. Simulation of a $2500 \times 2500-\mu$ m combined die-package system. (a) Voltage map of an on-chip layer (both VSS and VCC wires are shown). (b) Voltage map of the second on-chip layer (both VSS and VCC wires are shown). (c) Voltage map of a package VCC plane. (d) Voltage map of a package VSS plane. 


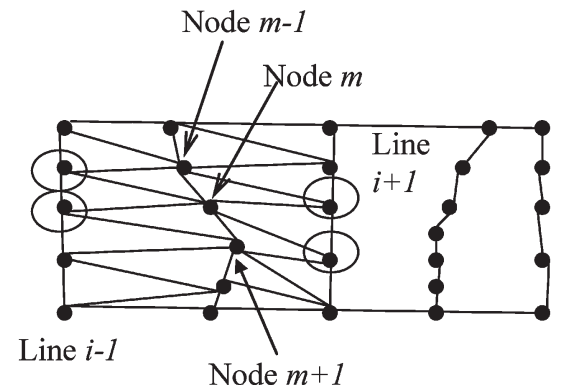

Fig. 17. Illustration of an arbitrary node $m$ and its connection with other nodes.

where

$$
\left\langle\xi_{m}, \xi_{n}\right\rangle_{\Omega}= \begin{cases}\frac{\Delta}{6}, & m=n \\ \frac{\Delta}{12}, & m \neq n\end{cases}
$$

in which $\Delta$ is the area of a triangular element $e$. Hence, $\mathbf{P}_{V l, m n}^{e}$ can be denoted by

$$
\mathbf{P}_{V l, m n}^{e} \sim \begin{cases}t, & m=n \\ t / 2, & m \neq n\end{cases}
$$

in which

$$
t=h_{l}\left(\mu_{0} \varepsilon_{l}^{e}+0.5 \Delta t \mu_{0} \sigma_{l}^{e}\right) \frac{\Delta}{6} .
$$

With the matrix elements of $\mathbf{P}_{V l}$ known, $\|(\mathbf{L}+\mathbf{U}) v\|$ and $\|\mathbf{P} v\|$ can be derived. Since $\mathbf{P}$ is tridiagonal and symmetric, $\|\mathbf{P} v\|$ can be written as

$$
\begin{aligned}
\|\mathbf{P} v\|=\sum_{m=1}^{M} \mid \mathbf{P}_{V l, m, m-1} v_{m-1} & +\mathbf{P}_{V l, m, m} v_{m} \\
& +\mathbf{P}_{V l, m, m+1} v_{m+1} \mid .
\end{aligned}
$$

Considering an arbitrary volume unknown denoted by node $m$ in Fig. 17, assuming that the mesh is reasonably good, and hence, the sizes of all the elements are similar, we obtain

$$
\mathbf{P}_{V l, m, m} \sim n_{e} t
$$

in which $n_{e}$ is the number of triangular elements that own node $m$ as one of their vertices. In (A.12), $\mathbf{P}_{V l, m, m}$ is obtained by assembling the contribution from $n_{e}$ elements. Based on our unknown ordering scheme, nodes $m-1, m$, and $m+1$ all reside on the same line, as shown in Fig. 17. Since nodes $m$ and $m-1$ can only be simultaneously shared by two elements and so are nodes $m$ and $m+1, \mathbf{P}_{V l, m, m-1}$ and $\mathbf{P}_{V l, m, m+1}$ are assembled from two elemental contributions as

$$
\begin{aligned}
& \mathbf{P}_{V l, m, m-1} \sim t \\
& \mathbf{P}_{V l, m, m+1} \sim t .
\end{aligned}
$$

Therefore, (A.11) can be written as

$$
\|\mathbf{P} v\|=\sum_{m=1}^{M}\left|(t) v_{m-1}+\left(n_{e} t\right) v_{m}+(t) v_{m+1}\right|
$$

Since nodes $m-1$ and $m+1$ are adjacent to node $m$, the eigenfield $v$ at these two nodes can be estimated by $v$ at node $m$.
Hence

$$
\|\mathbf{P} v\| \sim \sum_{m=1}^{M}\left|\left(n_{e}+2\right) t\right|\left|v_{m}\right|
$$

As shown in Fig. 17, node $m$ not only interacts with the adjacent two nodes residing on the same line but also interacts with nodes residing on the line left to it and the line right to it. The interaction with the nodes residing on the left line is characterized by $\mathbf{L}$ and that with the nodes residing on the right line is characterized by $\mathbf{U}$. Therefore, $\mathbf{L}$ and $\mathbf{U}$ consist of matrix elements $\mathbf{P}_{V l, m, n}$ in which node $n$ resides on the adjacent lines and connects directly to node $m$ through one edge. Assuming that the number of nodes $n$ is $n_{p},\|(\mathbf{L}+\mathbf{U}) v\|$ can be evaluated by

$$
\|(\mathbf{L}+\mathbf{U}) v\|=\sum_{m=1}^{M}\left|\sum_{n=1}^{n_{p}} \mathbf{P}_{V l, m n} v_{n}\right|=\sum_{m=1}^{M}\left|\sum_{n=1}^{n_{p}}(t) v_{n}\right| .
$$

Since the $n_{p}$ nodes are adjacent to node $m,(\mathrm{~A} .16)$ can be estimated as

$$
\|(\mathbf{L}+\mathbf{U}) v\| \sim \sum_{m=1}^{M} n_{p}|t|\left|v_{m}\right|
$$

Take the mesh shown in Fig. 17 as an example. $n_{p}=4$ (the number of circled nodes), and $n_{e}=6$; hence, from (A.15) and (A.17), $\|(\mathbf{L}+\mathbf{U}) v\|<\|\mathbf{P} v\|$.

There exists a general relationship between $n_{p}$ and $n_{e}$

$$
n_{e}=2\left(\frac{n_{p}}{2}+1\right)=n_{p}+2 .
$$

Hence, from (A.15) and (A.17), the following inequality always holds true:

$$
\|(\mathbf{L}+\mathbf{U}) v\|<\|\mathbf{P} v\|
$$

Therefore, from (A.5), $\rho(\mathbf{G})<1$. Hence, the iteration in (25) converges for any $b_{V l}$ and any initial vector $x^{(0)}$.

\section{ACKNOWLEDGMENT}

The authors would like to thank Dr. M. J. Kobrinsky and Dr. S. Chakravarty of Intel Corporation for providing the measured data and Dr. W. Shi of Intel Corporation for providing the package structures.

\section{REFERENCES}

[1] P. J. Restle, A. E. Ruehli, S. G. Walker, and G. Papadopoulos, "Full-wave PEEC time-domain method for the modeling of on-chip interconnects," IEEE Trans. Comput.-Aided Design Integr. Circuits Syst., vol. 20, no. 7, pp. 877-887, Jul. 2001.

[2] C. C. Chen, T. Lee, N. Murugesan, and S. C. Hagness, "Generalized FDTD-ADI: An unconditionally stable full-wave Maxwell's equations solver for VLSI interconnect modeling," in IEEE/ACM ICCAD, Nov. 2000, pp. 156-163.

[3] S. Kapur and D. E. Long, "Large-scale full-wave simulation," in 41th ACM/IEEE Des. Autom. Conf., 2004, pp. 806-809.

[4] A. Rong and A. C. Cangellaris, "Generalized PEEC models for threedimensional interconnect structures and integrated passives of arbitrary 
shapes," in IEEE 10th Top. Meet. Elect. Perform. Electron. Packag., Oct. 2001, pp. 225-228

[5] D. Gope, A. E. Ruehli, C. Yang, and V. Jandhyala, "(S)PEEC: timeand frequency-domain surface formulation for modeling conductors and dielectrics in combined circuit electromagnetic simulations," IEEE Trans. Microw. Theory Tech., vol. 54, no. 6, pp. 2453-2464, Jun. 2006.

[6] D. Jiao, C. Dai, S.-W. Lee, T. R. Arabi, and G. Taylor, "Computational electromagnetics for high-frequency IC design," in IEEE Int. Symp. Antennas Propag., 2004, pp. 3317-3320. invited paper.

[7] D. Jiao, M. Mazumder, S. Chakravarty, C. Dai, M. J. Kobrinsky, M. C. Harmes, and S. List, "A novel technique for full-wave modeling of large-scale three-dimensional high-speed on/off-chip interconnect structures," in Int. Conf. SISPAD, 2003, pp. 39-42.

[8] Z. H. Zhu, B. Song, and J. K. White, "Algorithm in FastImp: A fast and wideband impedance extraction program for complicated 3D geometries," in 40th ACM/IEEE Des. Autom. Conf., Jun. 2003, pp. 712-717.

[9] W. C. Chew, "Toward a more robust and accurate fast integral solver for microchip applications," in IEEE 12th Top. Meet. EPEP, 2003, p. 333.

[10] F. Ling, V. I. Okhamtovski, W. Harris, S. McCracken, and A. Dengi, "Large-scale broad-band parasitic extraction for fast layout verification of 3-D RF and mixed-signal on-chip structures," IEEE Trans. Microw. Theory Tech., vol. 53, no. 1, pp. 264-273, Jan. 2005.

[11] H. Gan and D. Jiao, "A fast and high-capacity electromagnetic solution for high-speed IC design," in IEEE/ACM ICCAD, 2007, pp. 1-6.

[12] H. Gan and D. Jiao, "A time-domain layered finite element reduction recovery (LAFE-RR) method for high-frequency VLSI design," IEEE Trans. Antennas Propag., vol. 55, no. 12, pp. 3620-3629, Dec. 2007.

[13] D. Jiao, J.-H. Kim, and J. He, "Efficient full-wave characterization of discrete high-density multiterminal decoupling capacitors for high-speed digital systems," IEEE Trans. Adv. Packag., vol. 31, no. 1, pp. 154-162, Feb. 2008

[14] M. J. Kobrinsky, S. Chakravarty, D. Jiao, M. C. Harmes, S. List, and M. Mazumder, "Experimental validation of crosstalk simulations for onchip interconnects at high frequencies using S-parameters," in IEEE 12th Top. Meet. EPEP, 2003, pp. 329-332.

[15] F. Sheng, S. Chakravarty, and D. Jiao, "An efficient 3D-to-2D reduction technique for frequency-domain layered finite element analysis of largescale high-frequency integrated circuits," in IEEE 16th Top. Meet. EPEP, Oct. 29-31, 2007, pp. 295-298.

[16] H. Gan and D. Jiao, "A fast-marching time-domain layered finite-element reduction-recovery method for high-frequency VLSI design," in IEEE IMS, Jun. 2008, pp. 169-172.

[17] W. Chai and D. Jiao, "An H-matrix-based method for reducing the complexity of integral-equation-based solutions of electromagnetic problems," in IEEE Int. Symp. Antennas Propag., Jul. 2008, pp. 1-4.

[18] J. Lee, V. Balakrishnan, C.-K. Koh, and D. Jiao, "A linear-time eigenvalue solver for finite-element-based analysis of large-scale wave propagation problems in on-chip interconnect structures," in IEEE Int. Symp. Antennas Propag., Jul. 2008, pp. 1-4.

[19] H. Gan and D. Jiao, "From $\mathrm{O}(\mathrm{N})$ to $\mathrm{O}(\mathrm{M})$ : time-domain layered finiteelement reduction-recovery methods for large scale electromagneticsbased analysis and design of on-chip circuits," in IEEE Int. Symp. Antennas Propag., Jul. 2008, pp. 1-4.

[20] D. Jiao, M. Lu, E. Michielssen, and J. M. Jin, "A fast time-domain finite element-boundary integral method for electromagnetic transient analysis," IEEE Trans. Antennas Propag., vol. 49, no. 10, pp. 1453-1461, Oct. 2001.

[21] D. Jiao and J. M. Jin, "Three-dimensional orthogonal vector basis functions for time-domain finite element solution of vector wave equations," IEEE Trans. Antennas Propag., vol. 51, no. 1, pp. 59-66, Jan. 2003.

[22] J. M. Jin, The Finite Element Method in Electromagnetics, 2nd ed. New York: Wiley, 2002. (442 p.).
[23] D. Jiao and J. M. Jin, "Finite element analysis in time domain," in The Finite Element Method in Electromagnetics. New York: Wiley, 2002, pp. 529-584.

[24] UMFPACK. [Online]. Available: http://www.cise.ufl.edu/research/sparse/ umfpack

[25] Y. Saad, Iterative Methods for Sparse Linear Systems, 2nd ed. Philadelphia, PA: SIAM, 2003

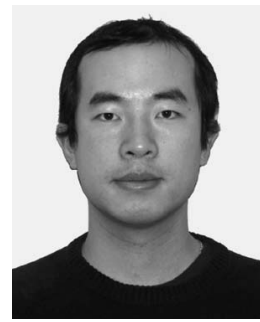

Duo Chen received the B.S. and M.S. degrees in electrical engineering from Tsinghua University, Beijing, China, in 2004 and 2007, respectively. He is currently working toward the Ph.D. degree in the School of Electrical and Computer Engineering, Purdue University, West Lafayette, IN.

$\mathrm{He}$ is also a Research Assistant with the On-Chip Electromagnetics Research Group, School of Electrical and Computer Engineering, Purdue University. His current research interest is the electromagneticsbased analysis of very large scale integration and

package problems

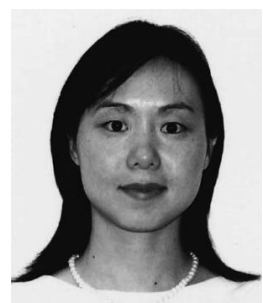

Dan Jiao (S'00-M'02-SM'06) received the Ph.D. degree in electrical engineering from the University of Illinois, Urbana-Champaign, in 2001.

She then worked with the Technology CAD Division, Intel Corporation, until September 2005 as a Senior CAD Engineer, Staff Engineer, and Senior Staff Engineer. Since September 2005, she has been with Purdue University, West Lafayette, IN, as an Assistant Professor with the School of Electrical and Computer Engineering. She has authored two book chapters and over 90 papers in refereed journals and international conferences. Her current research interests include high-frequency digital, analog, mixed-signal, and RF IC design and analysis, high-performance very large scale integration computer-aided design, modeling of micro- and nanoscale circuits, computational electromagnetics, applied electromagnetics, fast and high-capacity numerical methods, fast time-domain analysis, scattering and antenna analysis, RF, microwave, and millimeter-wave circuits, wireless communication, and bioelectromagnetics.

Dr. Jiao has served as a Reviewer for many IEEE journals and conferences. She was the recipient of the National Science Foundation CAREER Award in 2008. In 2006, she was the recipient of the Jack and Cathie Kozik Faculty Startup Award, which recognizes an outstanding new faculty member in Purdue Electrical and Computer Engineering. She also received an Office of Naval Research award through the Young Investigator Program. In 2004, she was the recipient of the Best Paper Award from Intel's annual corporate-wide technology conference (Design and Test Technology Conference) for her work on the generic broadband model of high-speed circuits. In 2003, she won the Intel Logic Technology Development (LTD) Divisional Achievement Award in recognition of her work on the industry-leading BroadSpice modeling/ simulation capability for designing high-speed microprocessors, packages, and circuit boards. She was also awarded the Intel Technology CAD Divisional Achievement Award for the development of innovative full-wave solvers for high-frequency IC design. In 2002, she was awarded by Intel Components Research the Intel Hero Award (Intel-wide, she was the tenth recipient) for the timely and accurate 2-D and 3-D full-wave simulations. She also won the Intel LTD Team Quality Award for her outstanding contribution to the development of the measurement capability and simulation tools for high-frequency on-chip crosstalk. She was the winner of the 2000 Raj Mittra Outstanding Research Award given her by the University of Illinois, Urbana-Champaign. 